\title{
Role of $\mathrm{Ag}^{+}$ions in determining $\mathrm{Ce}^{3+}$ Optical Properties in Fluorophosphate and Sulfophosphate Glasses
}

\author{
Ru Zhou, Courtney Calahoo, Yicong Ding, and Lothar Wondraczek* \\ Otto Schott Institute of Materials Research, Friedrich Schiller University Jena, \\ Fraunhoferstraße 6, 07743 Jena, Germany \\ *Email: lothar.wondraczek@uni-jena.de
}

This file contains supplementary Tables S1-S1 and Supplementary Figures S1-S10.

Table S1: Analyzed chemical compositions of the Ce-doped and Ag, Ce-codoped PF glass and PS glass series. The average error varies from $1-3 \mathrm{~mol} \%$.

\begin{tabular}{|c|c|c|c|c|c|c|}
\hline PF Samples & $\begin{array}{c}{\left[\mathrm{NaO}_{1 / 2}\right]} \\
(\mathrm{mol} \%)\end{array}$ & $\begin{array}{l}{\left[\mathrm{PO}_{2 / 5}\right]} \\
(\mathrm{mol} \%)\end{array}$ & $\begin{array}{l}{\left[\mathrm{AlF}_{3}+\right.} \\
\left.\mathrm{AlO}_{3 / 2}\right] \\
(\mathrm{mol} \%) \\
\end{array}$ & {$[\mathrm{F}] /[\mathrm{Al}]$} & $\begin{array}{c}{[\# \text { of } \mathrm{Na}} \\
\text { atoms }] /[100 \\
\text { atoms }]\end{array}$ & $\begin{array}{l}{[\# \text { of } \mathrm{O}} \\
\text { atoms]/[100 } \\
\text { atoms }]\end{array}$ \\
\hline PF5-Ce & 47.88 & 49.05 & 3.07 & 1.02 & 32.34 & 30.34 \\
\hline PF10-Ce & 44.71 & 49.62 & 5.67 & 1.00 & 29.73 & 29.74 \\
\hline PF15-Ce & 44.99 & 46.91 & 8.10 & 1.23 & 29.13 & 28.78 \\
\hline PF20-Ce & 43.51 & 45.89 & 10.60 & 1.35 & 27.47 & 27.78 \\
\hline PF5-Ag,Ce & 48.04 & 48.95 & 3.01 & 0.95 & 32.50 & 30.43 \\
\hline $\mathrm{PF} 10-\mathrm{Ag}, \mathrm{Ce}$ & 45.32 & 49.12 & 5.56 & 0.99 & 30.16 & 29.81 \\
\hline PF15-Ag,Ce & 44.17 & 47.63 & 8.20 & 1.06 & 28.80 & 29.12 \\
\hline $\mathrm{PF} 20-\mathrm{Ag}, \mathrm{Ce}$ & 43.36 & 45.81 & 10.83 & 1.23 & 27.50 & 28.06 \\
\hline PS Samples & $\begin{array}{c}{\left[\mathrm{NaO}_{1 / 2}\right]} \\
(\mathrm{mol} \%)\end{array}$ & $\begin{array}{l}{\left[\mathrm{PO}_{2 / 5}\right]} \\
(\mathrm{mol} \%)\end{array}$ & $\begin{array}{c}{\left[\mathrm{SO}_{3}\right]} \\
(\mathrm{mol} \%)\end{array}$ & $\begin{array}{c}{\left[\mathrm{AlO}_{3 / 2}\right]} \\
(\mathrm{mol} \%)\end{array}$ & $\begin{array}{c}{[\# \text { of } \mathrm{Na}} \\
\text { atoms }] /[100 \\
\text { atoms] }\end{array}$ & $\begin{array}{c}{[\# \text { of } \mathrm{O}} \\
\text { atoms }] /[100 \\
\text { atoms }]\end{array}$ \\
\hline PS5-Ce & 44.66 & 43.23 & 1.24 & 10.87 & 29.65 & 33.60 \\
\hline PS7.5-Ce & 45.68 & 41.40 & 1.98 & 10.94 & 29.93 & 34.48 \\
\hline PS10-Ce & 46.33 & 39.78 & 3.11 & 10.78 & 29.77 & 35.73 \\
\hline PS12.5-Ce & 47.05 & 38.52 & 3.81 & 10.62 & 29.88 & 36.49 \\
\hline PS15-Ce & 47.61 & 37.45 & 4.19 & 10.75 & 30.03 & 36.91 \\
\hline PS5-Ag,Ce & 44.65 & 43.24 & 1.32 & 10.79 & 29.61 & 33.68 \\
\hline PS7.5-Ag,Ce & 45.73 & 41.12 & 2.24 & 10.91 & 29.83 & 34.77 \\
\hline PS10-Ag,Ce & 46.52 & 40.15 & 2.67 & 10.66 & 30.12 & 35.25 \\
\hline PS12.5-Ag, Ce & 46.81 & 38.07 & 4.54 & 10.58 & 29.38 & 37.17 \\
\hline PS15-Ag,Ce & 47.89 & 37.02 & 4.43 & 10.66 & 30.09 & 37.22 \\
\hline
\end{tabular}


Table S2: Density $\rho$, molar volume $V_{m}$, glass transition temperature $T_{g}$, Young's modulus E, Poisson ratio $v$ and optical basicity $\Lambda_{\text {th }}$ of studied glasses.

\begin{tabular}{|c|c|c|c|c|c|c|c|c|}
\hline \multirow[t]{2}{*}{ Glass } & \multirow{2}{*}{$\begin{array}{c}\text { Density } \\
\left(\mathrm{g} / \mathrm{cm}^{3}\right) \\
( \pm 0.001)\end{array}$} & \multirow{2}{*}{$\begin{array}{c}\mathrm{V}_{\mathrm{m}} \\
\left(\mathrm{cm}^{3} / \mathrm{mol}\right)\end{array}$} & \multirow{2}{*}{$\begin{array}{l}\text { Refractive } \\
\text { index } n_{\mathrm{d}} \\
( \pm 0.0005)\end{array}$} & \multirow{2}{*}{$\begin{array}{c}T_{\mathrm{g}}\left({ }^{\circ} \mathrm{C}\right) \\
( \pm 2)\end{array}$} & \multirow{2}{*}{$\begin{array}{c}E(\mathrm{GPa}) \\
( \pm 0.7)\end{array}$} & \multirow{2}{*}{$\begin{array}{c}v \\
( \pm 0.003)\end{array}$} & \multicolumn{2}{|c|}{$\Lambda_{\text {th }}$} \\
\hline & & & & & & & Nom. & Anal. \\
\hline PF5-0.5Ce & 2.550 & 39.854 & 1.516 & 293.0 & 39.9 & 0.291 & 0.455 & 0.454 \\
\hline PF5-0.5Ag-0.5Ce & 2.558 & 39.861 & 1.517 & 287.4 & 39.6 & 0.283 & & \\
\hline PF10-0.5Ce & 2.583 & 38.997 & 1.509 & 314.2 & 42.3 & 0.287 & 0.440 & 0.449 \\
\hline PF $10-0.5 \mathrm{Ag}-0.5 \mathrm{Ce}$ & 2.587 & 39.070 & 1.511 & 312.3 & 42.7 & 0.283 & & \\
\hline PF15-0.5Ce & 2.614 & 38.192 & 1.508 & 333.5 & 45.3 & 0.282 & 0.425 & 0.447 \\
\hline PF $15-0.5 \mathrm{Ag}-0.5 \mathrm{Ce}$ & 2.617 & 38.281 & 1.510 & 337.0 & 46.2 & 0.283 & & \\
\hline PF20-0.5Ce & 2.629 & 37.633 & 1.507 & 369.9 & 52.7 & 0.275 & 0.410 & 0.439 \\
\hline PF20-0.5Ag-0.5Ce & 2.639 & 37.781 & 1.510 & 370.7 & 52.7 & 0.275 & & \\
\hline PS5-0.5Ce & 2.645 & 39.502 & 1.520 & 413.6 & 52.1 & 0.282 & 0.4638 & 0.4525 \\
\hline PS5-0.5Ag-0.5Ce & 2.661 & 39.476 & 1.521 & 418.1 & 52.9 & 0.280 & & \\
\hline PS7.5-0.5Ce & 2.646 & 39.880 & 1.521 & 414.6 & 51.4 & 0.286 & 0.4641 & 0.4579 \\
\hline PS7.5-0.5Ag-0.5Ce & 2.652 & 39.910 & 1.523 & 416.9 & 51.4 & 0.280 & & \\
\hline PS10-0.5Ce & 2.643 & 40.304 & 1.524 & 414.8 & 50.6 & 0.283 & 0.4645 & 0.4583 \\
\hline PS10-0.5Ag-0.5Ce & 2.652 & 40.285 & 1.525 & 411.6 & 50.1 & 0.287 & & \\
\hline PS12.5-0.5Ce & 2.637 & 40.774 & 1.526 & 412.6 & 49.6 & 0.292 & 0.4649 & 0.4585 \\
\hline PS12.5-0.5Ag-0.5Ce & 2.650 & 40.691 & 1.526 & 414.4 & 49.9 & 0.290 & & \\
\hline PS15-0.5Ce & 2.635 & 41.185 & 1.527 & 410.6 & 48.2 & 0.293 & 0.4652 & 0.4589 \\
\hline PS15-0.5Ag-0.5Ce & 2.642 & 41.191 & 1.528 & 417.9 & 48.1 & 0.291 & & \\
\hline
\end{tabular}
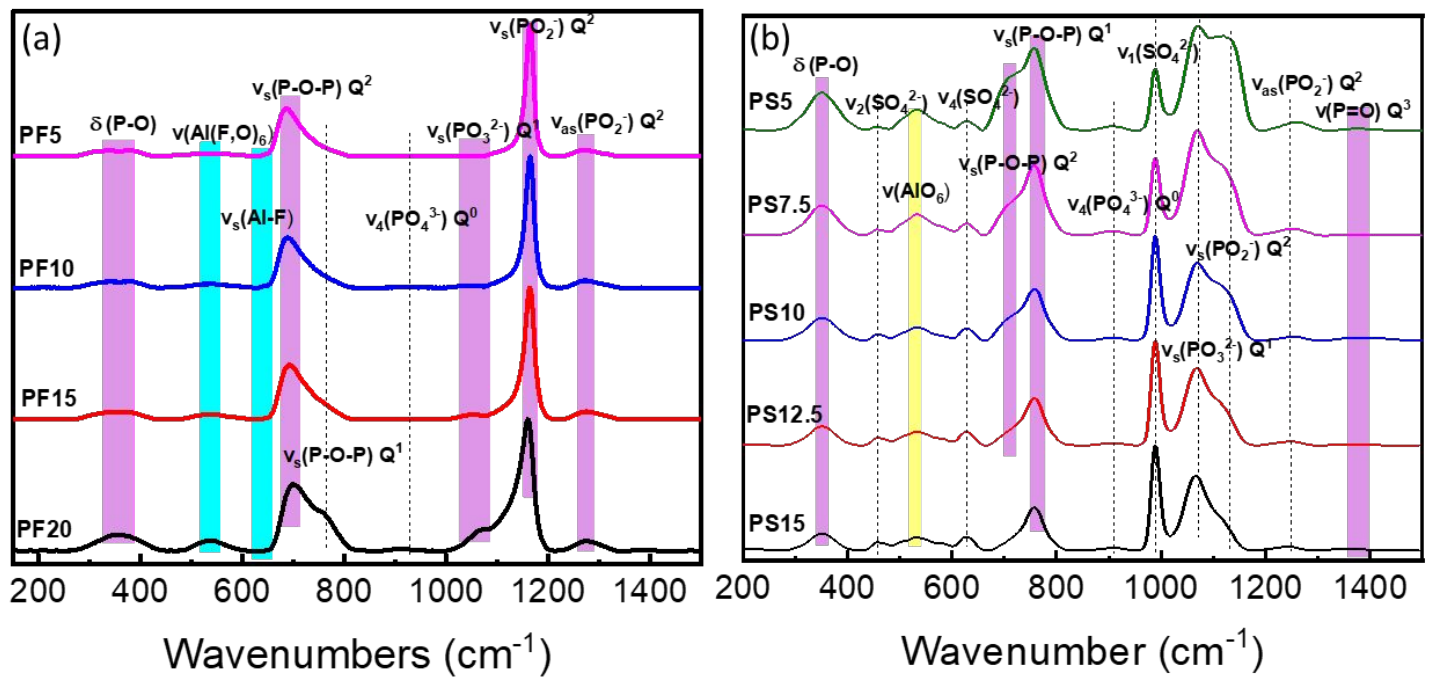

Figure S1:Raman spectra of Ce-doped (a) PF glasses and (b) PS glasses . 

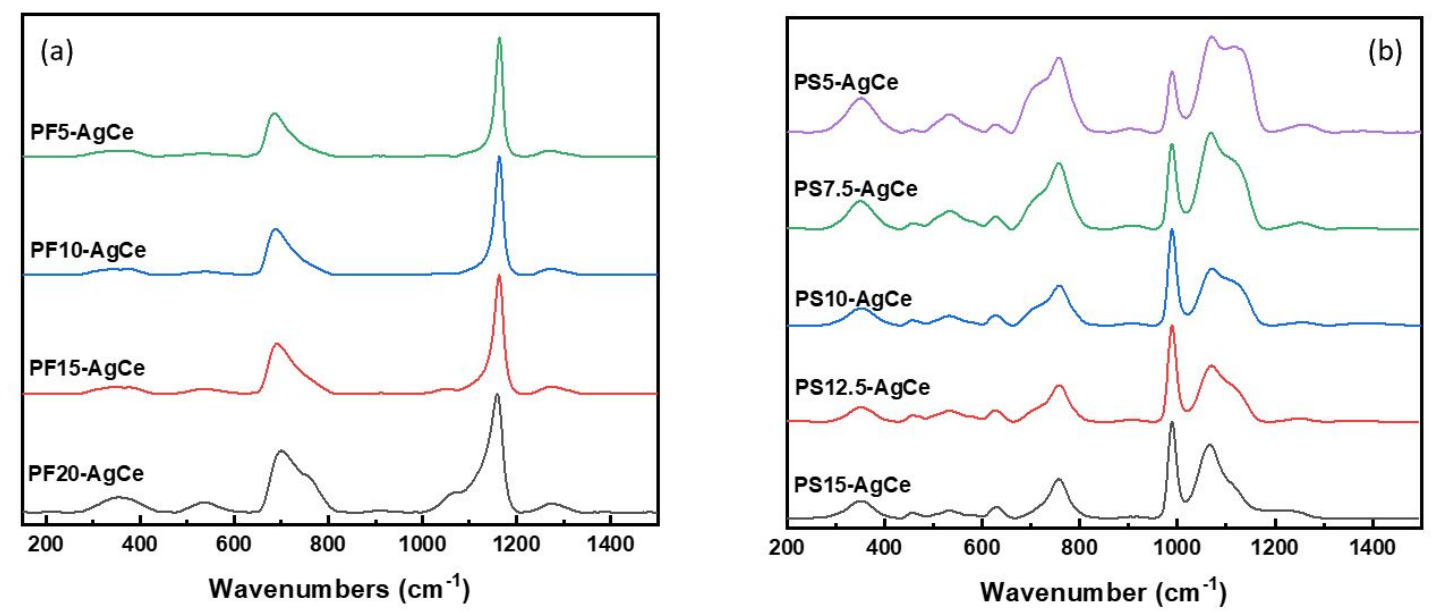

Figure S2:Raman spectra of AgCe-codoped (a) PF glasses and (b)PS glasses .
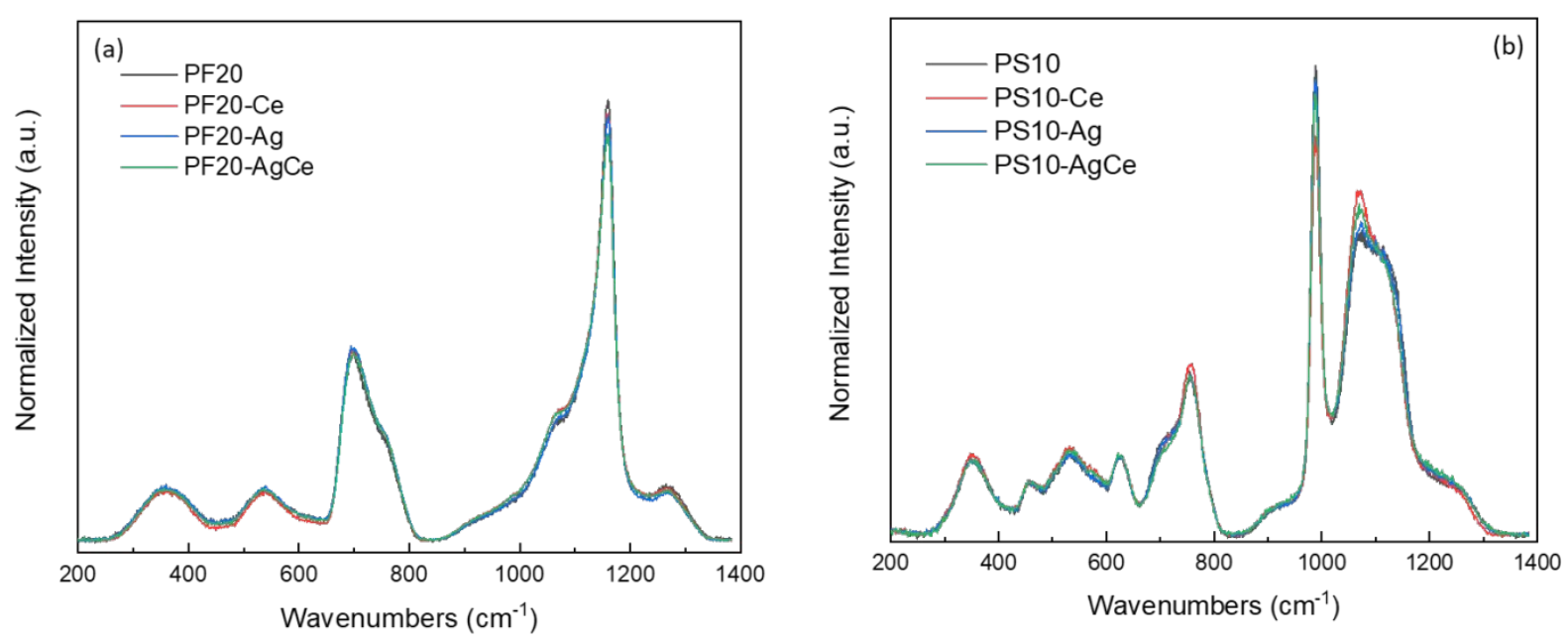

Figure S3:Normalized Raman spectra of (a) PF20 glasses and (b) PS10 glasses with different dopants. 


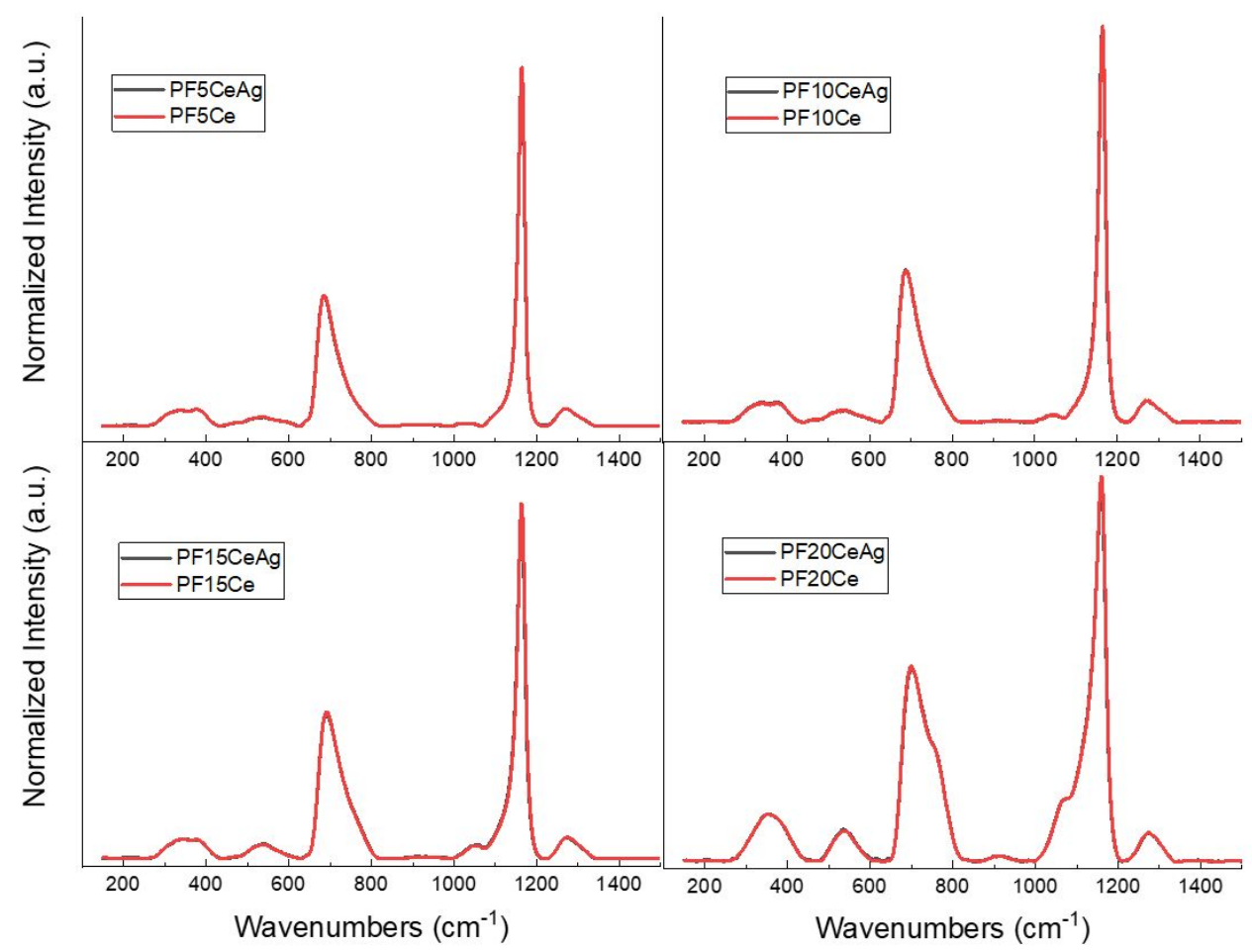

Figure S4:Normalized Raman spectra of PF glasses with single Ce and AgCe-codoping.

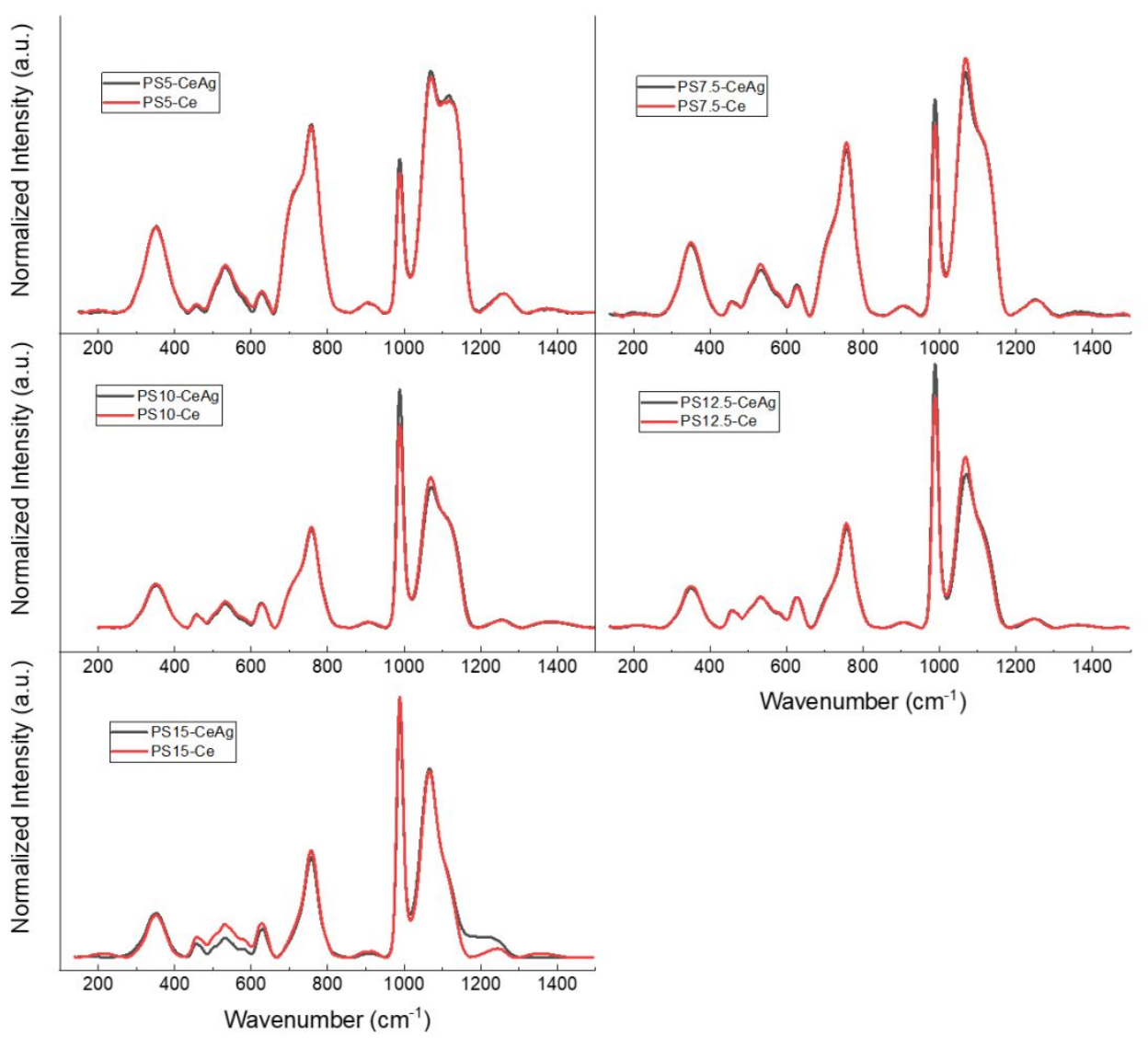

Figure S5: Normalized Raman spectra of PS glasses with single Ce and AgCe-codoping. 

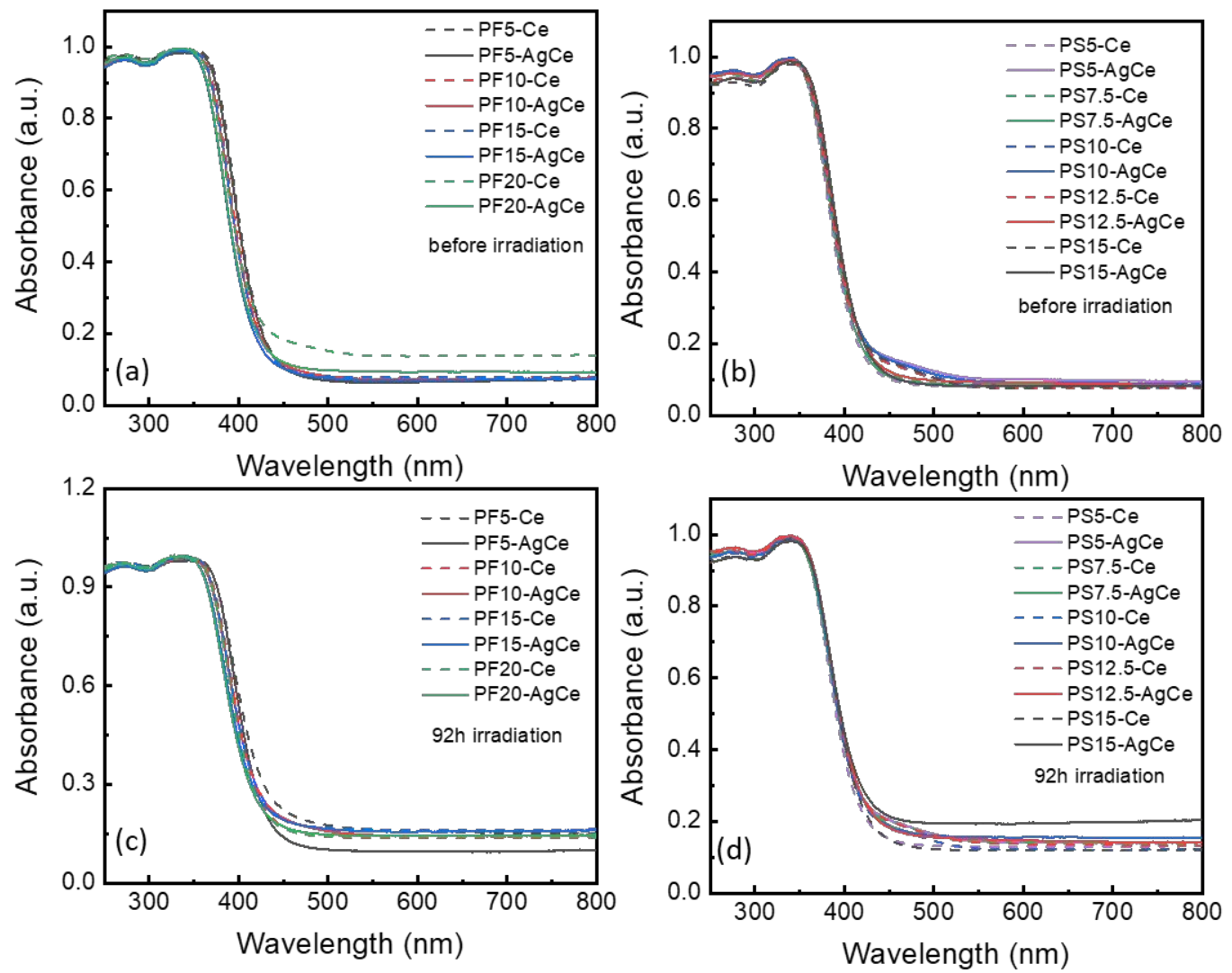

Figure S6:Absorption spectra of Ce-doped and Ag, Ce-codoped (a) PF glasses and (b)PS glasses before irradiation; the absorption spectra of Ce-doped and Ag, Ce-codoped (c) PF glasses and (d)PS glasses after irradiation.
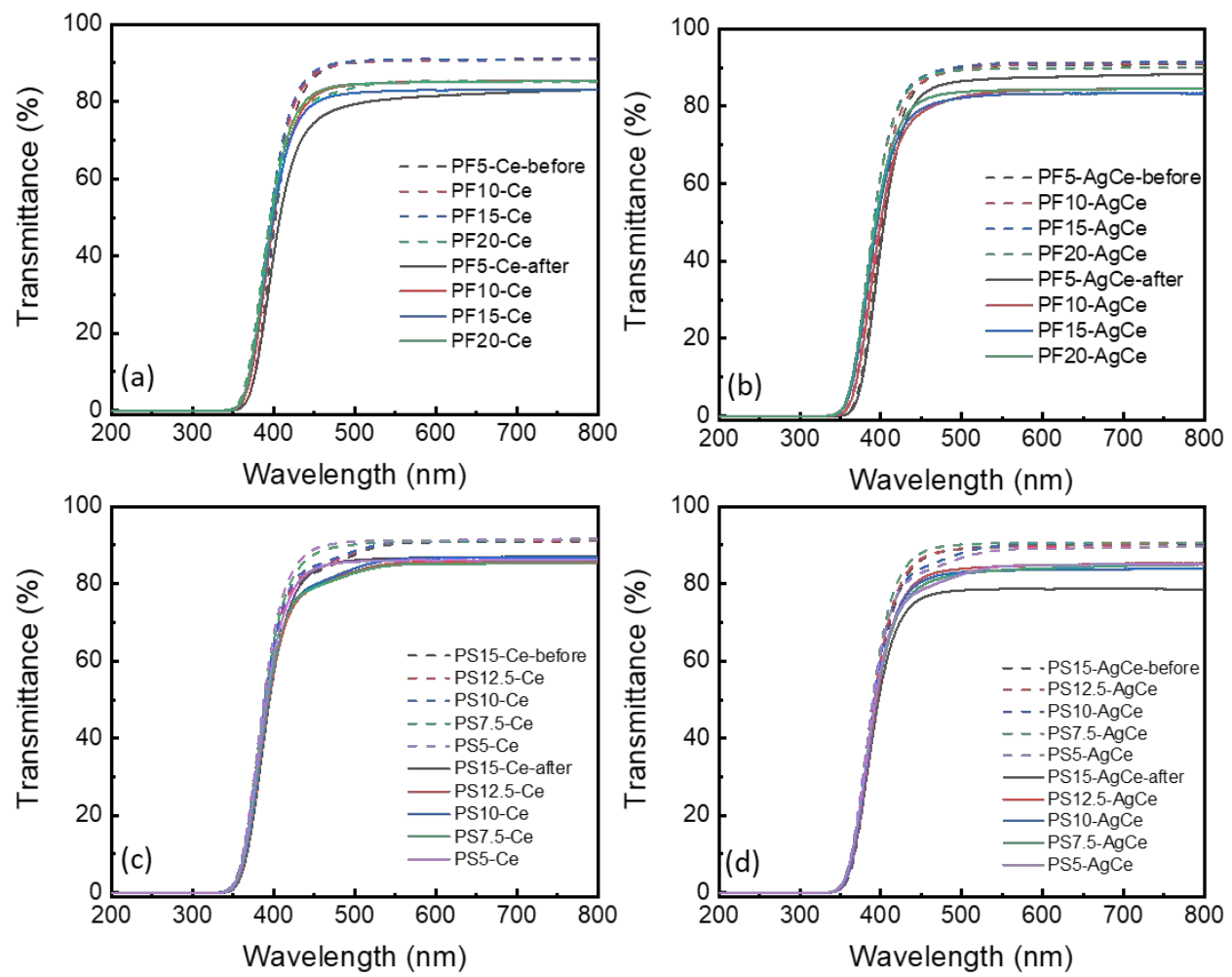

Figure S7:Transmittance spectra of (a) Ce-doped PF glasses, (b) Ag, Ce-codoped PF glasses, (c) Ce-doped PS glasses, and (d) Ag, Ce-codoped PS glasses before and after irradiation. 

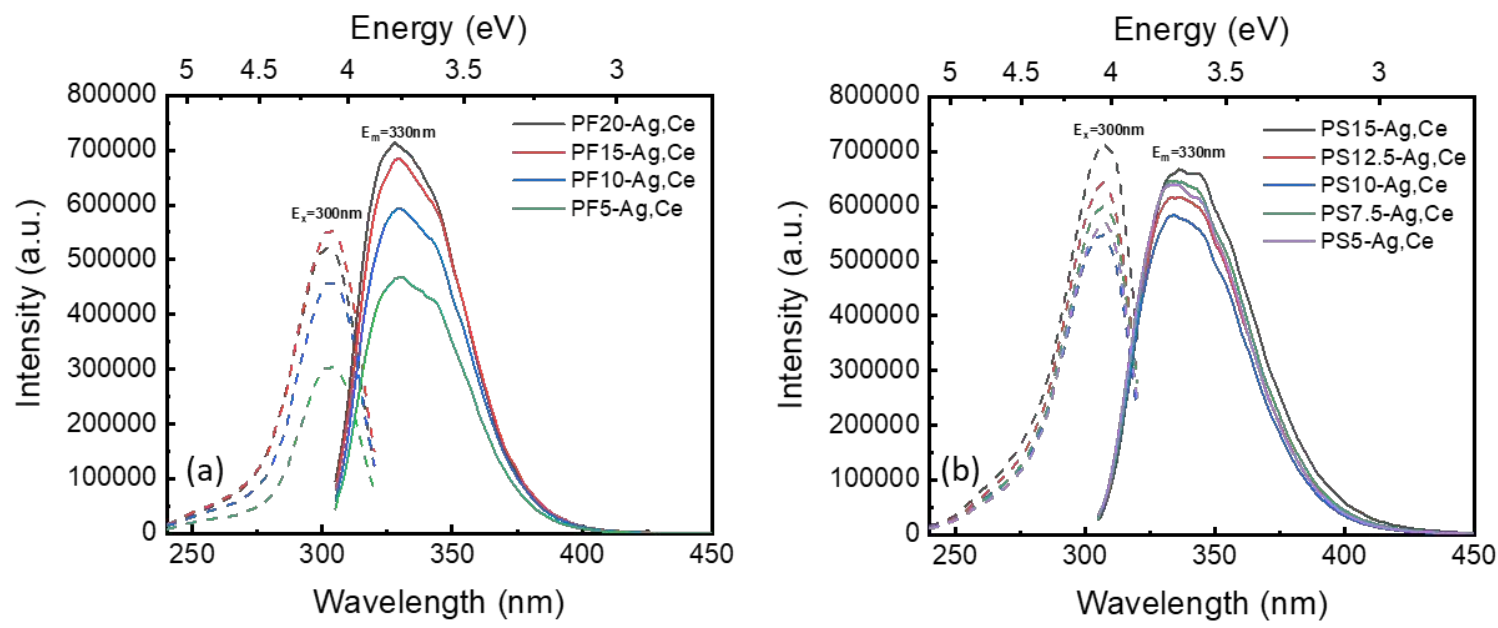

Figure S8: PLE and PL spectra of (a) Ag, Ce-doped PF glasses and (b) Ag, Ce-doped PS glasses under $\mathbf{E}_{\mathrm{x}}=300 \mathrm{~nm}$.
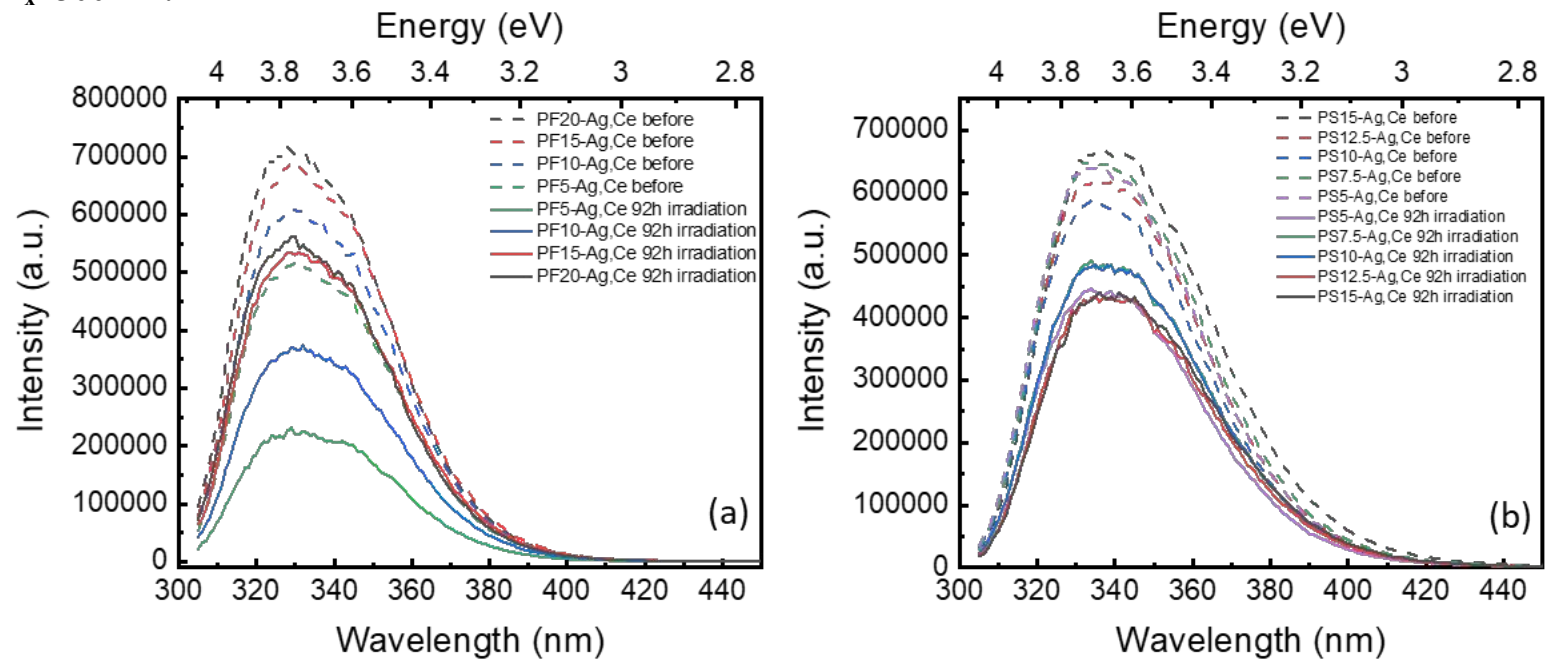

Figure S9: PL spectra of Ag, Ce-codoped (a) PF glasses and (b)PS glasses before and after irradiation under $\mathbf{E}_{\mathbf{x}}=300 \mathrm{~nm}$.
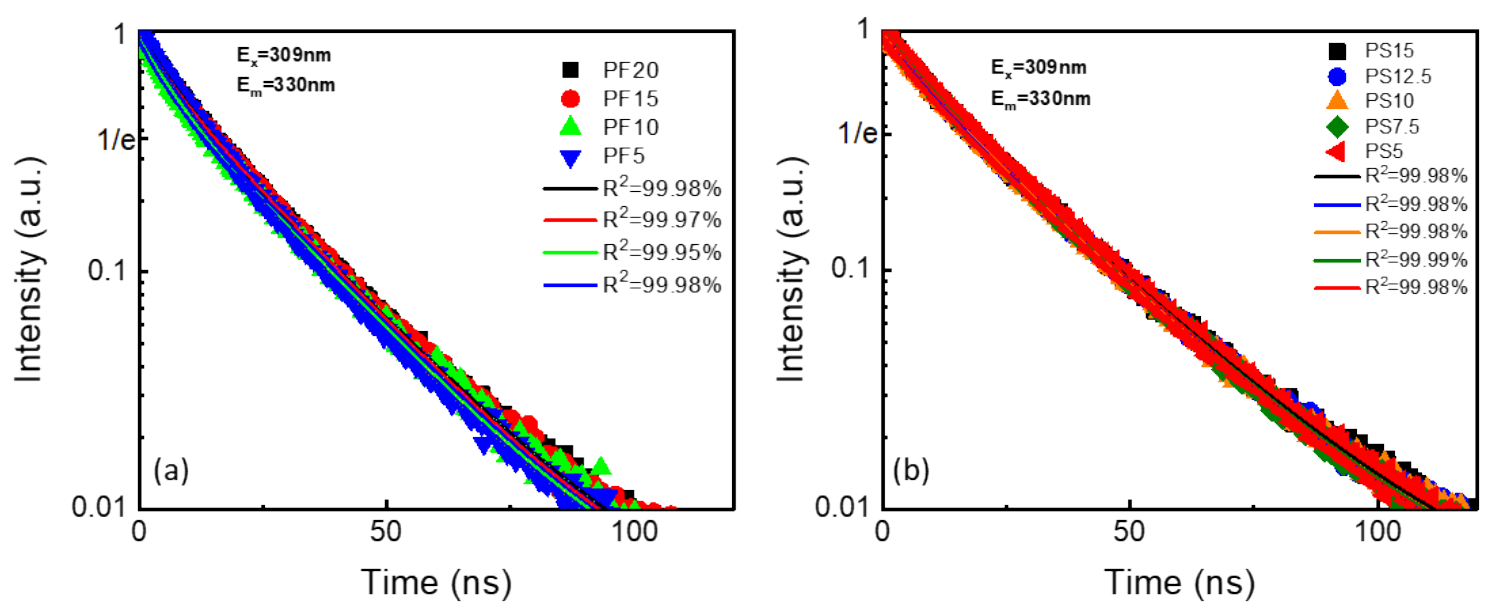

Figure S10: Luminescence decay curves of (a) Ce-doped PF glasses and (b) Ce-doped PS glasses (excitation and emission wavelengths as labelled). 